\title{
THE COMBINED EFFECTS OF POSITIVE ASSORTATIVE MATING AND SELECTION
}

\author{
CATHERINE T. FALK \\ Department of Serology and Genetics, The New York Blood Center,
}

Received 2. ix.70

\section{INTRODUGTION}

Posirrve assortative mating has been observed in natural populations of a variety of organisms. In man Pearson and Lee (1903) demonstrated evidence of positive assortative mating for certain physical characteristics. Other examples have been described for the blue snow goose (Cooch and Beardmore, 1959), the Artic Skua (O'Donald, 1959), and Drosophila melanogaster (Parsons, 1965). Laboratory experiments have been carried out to observe the consequences of induced positive assortative mating (Breese, 1956; Thoday, 1959). One such consequence was an increase in genetic variability. These experimental observations raise a number of questions: What effect does positive assortative mating have on polymorphisms present in nature? Can balanced polymorphisms be maintained when positive assortative mating is taking place? What is the effect of the combination with other genetic forces, such as selection?

Some answers have been provided by the study of theoretical models. O'Donald (1960) set up a model of mixed random mating and positive assortative mating. He studied the equilibrium behaviour of the model and obtained an expression for the heterozygote frequency as a function of the generation time, the initial and equilibrium heterozygote frequencies and the gene frequencies. Parsons (1962) considered the same model as O'Donald but expanded it to include the influence of selection on the genotypes. Rather than studying the equilibrium behaviour Parsons then considered conditions under which new genes would increase in frequency in a population. This enabled him to use approximations to the difference equations describing the model, thus simplifying the algebraic manipulation necessary.

Two recent companion papers (Scudo and Karlin, 1969; Karlin and Scudo, 1969) examine a series of models of assortative mating in which preference is exercised by only one sex. They point out that in previous work the model used was such that gene frequencies remained constant and only the genotype frequencies changed with time.

The present paper considers the same model as that described by Parsons (1962) and studies the equilibrium behaviour of a population in which the three possible genotypes are all present in the population. With the presence of selective pressures, the gene frequencies as well as the genotype frequencies will change with time until an equilibrium is reached.

\section{Assortative mating; one allele dominant}

Let $u, v$ and $w$ be the frequencies of three genotypes $A A, A a$ and $a a$ in a population. Assume that a fraction $\alpha$ of the population mates with like phenotypes and the remainder $1-\alpha$ mates at random. If gene $A$ is com- 
pletely dominant to $a$, the genotypes $A A$ and $A a$ will be phenotypically indistinguishable. The mating types and their frequencies for the assortative

TABLE 1

Frequency of mating types; positive assortative mating with dominance

\begin{tabular}{|c|c|c|c|c|}
\hline & $A A$ & $A a$ & $a a$ & \\
\hline$A A$ & $u^{2} /(u+v)$ & $u v /(u+v)$ & 0 & $u$ \\
\hline$A a$ & $u v /(u+v)$ & $v^{2} /(u+v)$ & 0 & $v$ \\
\hline$a a$ & 0 & 0 & $w$ & $w$ \\
\hline & $u$ & $v$ & $w$ & 1 \\
\hline
\end{tabular}

mating fraction of the population are given in Table 1. The total frequencies of the six mating types will then be:

\begin{tabular}{|c|c|c|c|}
\hline \multirow[b]{2}{*}{ Mating type } & \multicolumn{3}{|c|}{ Frequency } \\
\hline & assortative & & random \\
\hline$A A \times A A$ & $\alpha \cdot \frac{u^{2}}{(u+v)}$ & + & $(1-\alpha) u^{2}$ \\
\hline$A A \times A a$ & $\alpha \cdot \frac{2 u v}{(u+v)}$ & + & $2(1-\alpha) u v$ \\
\hline$A a \times A a$ & $a \cdot \frac{v^{2}}{(u+v)}$ & + & $(1-a) v^{2}$ \\
\hline$A A \times a a$ & - & & $2(1-\alpha) u w$ \\
\hline$A a \times a a$ & - & & $2(1-\alpha) v w$ \\
\hline$a a \times a a$ & $\alpha \cdot w$ & + & $(1-\alpha) w^{2}$ \\
\hline Total & $\alpha$ & + & $(1-\alpha)$ \\
\hline
\end{tabular}

Let the relative viabilities of $A A, A a$ and $a a$ be $a, h$, and $b$ respectively. The frequencies of the three genotypes in the next generation, denotcd by $u^{\prime}, v^{\prime}$ and $w^{\prime}$ will be given by:

$$
\begin{aligned}
T u^{\prime} & =a\left[\frac{\alpha\left(u^{2}+u v+\frac{1}{4} v^{2}\right)}{u+v}+(1-\alpha)\left(u^{2}+u v+\frac{1}{4} v^{2}\right)\right] \\
& =a\left(u+\frac{1}{2} v\right)^{2}\left[\frac{\alpha}{u+v}+(1-\alpha)\right], \\
T v^{\prime} & =h\left[\frac{\alpha v}{u+v}\left(u+\frac{1}{2} v\right)+2(1-\alpha)\left(u+\frac{1}{2} v\right)\left(\frac{1}{2} v+w\right)\right] \\
& =h\left(u+\frac{1}{2} v\right)\left[\frac{\alpha v}{u+v}+2(1-\alpha)\left(\frac{1}{2} v+w\right)\right],
\end{aligned}
$$




$$
T w^{\prime}=b\left[\alpha w+\frac{\alpha v^{2}}{4(u+v)}+(1-\alpha)\left(\frac{1}{2} v+w\right)^{2}\right],
$$

where $T$ is defined so that $u^{\prime}+v^{\prime}+w^{\prime}=1$. These equations are identical to Parsons' equations (2.1)-(2.3) (1962), and O'Donald's model (1960) is the special case where $a=h=b=1$.

The question to be considered now is what is the nature of equilibrium states of this model if all three genotypes are present in the population? When are the equilibria stable and what is the role of selection in classifying the equilibria? Consider first a special case.

Complete assortative mating. For complete assortative mating $\alpha=1$ and equations (1)-(3) take on the form:

$$
\begin{aligned}
T u^{\prime} & =\frac{a\left(u+\frac{1}{2} v\right)^{2}}{u+v}, \\
T v^{\prime} & =\frac{h v\left(u+\frac{1}{2} v\right)}{u+v}, \\
T w^{\prime} & =\frac{b\left[4 w(u+v)+v^{2}\right]}{4(u+v)} .
\end{aligned}
$$

Assume $u, v, w \neq 0$. At equilibrium $u=u^{\prime}, v=v^{\prime}$ and $w=w^{\prime}$. The ratio of equation $\left(1^{\prime}\right)$ to $\left(2^{\prime}\right)$ at equilibrium gives:

$$
\frac{u}{v}=\frac{a}{2(h-a)} \equiv k_{1} \text {. }
$$

Making the substitution $u=k_{1} v$ in the ratio of equation $\left(1^{\prime}\right)$ to $\left(3^{\prime}\right)$, a linear equation in $v$ is obtained. The simplified result is:

where

$$
\hat{v}=\frac{2(h-a) M}{D},
$$

and

$$
\begin{aligned}
M & =h^{2}-b(2 h-a) \\
D & =h[(2 h-a)(h-2 b)+b h] .
\end{aligned}
$$

Since $\hat{u}=k_{1} \hat{t}$, and $\hat{w}=1-\hat{u}-\hat{v}$, there is an equilibrium point:

$$
\begin{aligned}
& \hat{u}=\frac{a M}{D}, \\
& \hat{v}=\frac{2(h-a) M}{D}, \\
& \hat{v}=\frac{b(h-a)^{2}}{D} .
\end{aligned}
$$

In order to have a genetic equilibrium with non-zero frequencies for the three genotypes, the following inequalities must be satisfied:

$$
\begin{aligned}
& 0<\frac{a M}{D}<1, \\
& 0<\frac{2(h-a) M}{D}<1, \\
& 0<\frac{b(h-a)^{2}}{D}<1 .
\end{aligned}
$$


All will be satisfied if the following relationships obtain:

$$
h>a, h>b, \frac{h}{b}>\frac{h-a}{h-b},
$$

as may be seen from the relations

$$
\begin{aligned}
M & =h(h-b)-b(h-a), \\
D & =h[M+(h-a)(h-b)], \\
D-a M & =(h-a)[M+h(h-b)], \\
D-2(h-a) M & =a M+b(h-a)^{2},
\end{aligned}
$$

If the restriction $u, v, w \neq 0$ is removed, certain "boundary" equilibria exist, where one or two of the genotype frequencies will be zero.

Local stability conditions. Equations $\left(1^{\prime}\right)-\left(3^{\prime}\right)$ can be rewritten in the form:

and

$$
\begin{aligned}
u^{\prime} & =f(u, v), \\
v^{\prime} & =g(u, v), \\
w^{\prime} & =1-u^{\prime}-v^{\prime} .
\end{aligned}
$$

These can be expanded about the known equilibrium point to give:

and

$$
f(u, v)=f(\hat{u}, \hat{v})+(u-\hat{u}) f_{u}+(v-\hat{v}) f_{v}+\ldots
$$

where

$$
g(u, v)=g(\hat{u}, \hat{v})+(u-\hat{u}) g_{u}+(v-\hat{v}) g_{v}+\ldots
$$

$$
f_{u}=\frac{\partial f}{\partial u} \mid \begin{aligned}
& u=\hat{u} \\
& v=\hat{v}
\end{aligned} \quad \text { etc. }
$$

If the population is close to the equilibrium point, the higher order terms of the expansion may be neglected. Now

and

$$
f(\hat{u}, \hat{v})=\hat{u}
$$

$$
g(\hat{u}, \hat{v})=\hat{v} \text {. }
$$

For generality replace $u^{\prime}$ with $u_{n+1}, u$ with $u_{n}$, etc, where the subscripts denote successive generations for a given value of $n$, and let $x_{n}=u_{n}-\hat{u}$, $y_{n}=v_{n}-\hat{v}$.

Then in the neighbourhood of equilibrium (4) and (5) can be approximated by:

$$
\begin{aligned}
& \text { and } \begin{array}{l}
u_{n+1}-\hat{u}=x_{n+1}=x_{n} f_{u}+y_{n} f_{v}, \\
v_{n+1}-\hat{v}=y_{n+1}=x_{n} g_{u}+y_{n} g_{v} .
\end{array} \\
& \text { Let } \boldsymbol{V}_{n}=\left(\begin{array}{l}
x_{n} \\
y_{n}
\end{array}\right) \\
& \text { and } \\
& G=\left(\begin{array}{ll}
f_{u} & f_{v} \\
g_{u} & g_{v}
\end{array}\right)
\end{aligned}
$$


Now (6) and (7) can be written

$$
\boldsymbol{V}_{n+1}=G \boldsymbol{V}_{n}
$$

the solution being

$$
\boldsymbol{V}_{n}=G^{n} \boldsymbol{V}_{\boldsymbol{o}}
$$

For a unique solution require:

$$
\left|\begin{array}{ll}
f_{u} & f_{v} \\
g_{u} & g_{v}
\end{array}\right| \neq 0
$$

In Appendix A the partial derivatives of $f$ and $g$ have been computed. The requirement for uniqueness is merely $a \neq 2 h$. In considering the internal genetic equilibrium with $u, v, w \neq 0$, this is always fulfilled since $h>a$.

The matrix $G$ can be diagonalised by a similarity transformation so that

$$
S^{-1} G S=\left(\begin{array}{cc}
\lambda_{1} & 0 \\
0 & \lambda_{2}
\end{array}\right) \equiv G_{d}
$$

where $\lambda_{1}$ and $\lambda_{2}$ are the eigenvalues of $G$, and the columns of $S$ are the righthand eigenvectors of $G$. $G^{n}$ can then be written

$$
G^{n}=S\left(G_{d}\right)^{n} S^{-1}=S\left(\begin{array}{cc}
\lambda_{1}^{n} & 0 \\
0 & \lambda_{2}^{n}
\end{array}\right) S^{-1}
$$

Equation (8) thus becomes

$$
\boldsymbol{V}_{n}=S\left(\begin{array}{cc}
\lambda_{1}^{n} & 0 \\
0 & \lambda_{2}^{n}
\end{array}\right) S^{-1} V_{0} .
$$

For a stable equilibrium $x_{n}$ and $y_{n}$ must approach zero as $n$ approaches infinity. This will be satisfied when $\left|\lambda_{1}\right|$ and $\left|\lambda_{2}\right|$ are less than one. The solution of the characteristic equation gives, after algebraic simplification:

and

$$
\begin{aligned}
& \lambda_{1}=\frac{a}{h} \\
& \lambda_{2}=\frac{b(2 h-a)}{h^{2}} .
\end{aligned}
$$

For the internal equilibrium being considered $\left|\lambda_{1}\right|<1$ is always satisfied. $\left|\lambda_{2}\right|<1$ implies that

$$
b(2 h-a)<h^{2}
$$

or, after rearrangement,

$$
\frac{h}{b}>\frac{h-a}{h-b} .
$$

This condition is also satisfied for the internal equilibrium. Hence by fulfilling the conditions for a genetic equilibrium with $u, v, w \neq 0$, the conditions for local stability of the equilibrium are also fulfilled. 
Partial assortative mating. Make the substitutions

and

$$
\begin{aligned}
\bar{T} & =(u+v) T, \\
p & =u+\frac{1}{2} v, \\
1-\mathrm{p} & =w+\frac{1}{2} v
\end{aligned}
$$

in equations (1)-(3). The rewritten equations will then be in terms of the two variables $p$ and $v$ with $\bar{T}$ defined by the normalising restriction

$u^{\prime}+v^{\prime}+w^{\prime}=1$. Then equation (1) plus one-half of equation (2) gives:

$$
\bar{T} p^{\prime}=a p^{2}\left[\alpha+(1-\alpha)\left(p+\frac{1}{2} v\right)\right]+\frac{1}{2} h p\left[\alpha v+2(1-\alpha)(1-p)\left(p+\frac{1}{2} v\right)\right] .
$$

This together with equation (2) rewritten in terms of $p$ and $v$ :

$$
\tau_{v^{\prime}}=h p\left[\alpha v+2(1-\alpha)(1-p)\left(p+\frac{1}{2} v\right)\right]
$$

and the normalising equation, give the necessary independent equations for obtaining equilibrium frequencies for both the genes and the genotypes. The algebra involved in obtaining these equilibria is cumbersome and the equilibrium values themselves are so complicated that they are of little practical use. The end-results will be included for completeness but the intermediate steps will be omitted. Details of these steps can be found in Falk (1968).

Let $\epsilon=h-a$ and $\delta=h-b$. Then equation (9) and the equation defining $\tilde{T}$ lead to

where

$$
v B(p)=2 p(1-p) A(p)
$$

and

$$
\begin{aligned}
& A(p)=\delta-a \epsilon-p(\epsilon+\delta)(1-\alpha) \\
& B(p)=p A(p)+p(\epsilon+\delta)-\delta .
\end{aligned}
$$

For $B(p) \neq 0$, the expression for $v$ in terms of $p$ may be substituted into equation (10) and the resulting quadratic solved for $p$. This leads to

where

$$
p=\frac{1}{2}+\frac{1}{4}\left[\eta \pm \sqrt{\eta^{2}+4 h r}\right] \frac{\delta-\epsilon}{r},
$$

and

$$
\begin{aligned}
\eta & =(1+\alpha) \epsilon b-h(\delta+\epsilon) \\
r & =\epsilon b(\epsilon+\delta)(1-\alpha) \neq 0 .
\end{aligned}
$$

For the special case $a=b$ and therefore $\delta=\epsilon$, the equilibrium value for $p$ is $\frac{1}{2}$. Substitution into equation (11) gives no corresponding value for $v$ since $A(p)$ and $B(p)$ are then both zero. By substitution of $p=\frac{1}{2}$ and $b=a$ in equation (10), the corresponding equilibrium value for $v$ is obatined:

where

$$
v=\frac{C+\sqrt{C^{2}+4 h(1-\alpha) Q}}{2 Q},
$$

and

$$
C=2 \alpha h-a(1+\alpha)
$$$$
Q=a(1-\alpha)+h(1+\alpha) \text {. }
$$

In principle, the equilibrium value for $v$ for the general case, $\delta \neq \epsilon$, can be obtained by substituting the value of $p$ given by equation (12) into equation (11), but this is neither practical nor useful. For particular values of the fitness parameters the numerical value of $v$ can easily be obtained. 


\section{Assortative mating; No Dominance}

If niether allele $A$ nor allele $a$ is dominant, all three genotypes will be distinct with respect to the characteristic influencing mating choice and the corresponding mathematical model is somewhat modified. The recurrence equations will be:

$$
\begin{aligned}
T u^{\prime} & =a\left[\alpha\left(u+\frac{1}{4} v\right)+(1-\alpha)\left(u+\frac{1}{2} v\right)^{2}\right], \\
T v^{\prime} & =h\left[\frac{1}{2} \alpha v+2(1-\alpha)\left(u+\frac{1}{2} v\right)\left(\frac{1}{2} v+w\right)\right], \\
T w^{\prime} & =b\left[\alpha\left(\frac{1}{4} v+w\right)+(1-\alpha)\left(\frac{1}{2} v+w\right)^{2}\right],
\end{aligned}
$$

with all symbols defined as before.

The special case $\alpha=1$ gives results analogous to a selfing population where no mating takes place between different genotypes. This results in the reduction of the heterozygote frequency each generation unless the fitness of the heterozygote is more than double the fitness of each of the homozygotes (Hayman and Mather, 1953). If $h>2 a$ and $h>2 b$, then a sort of equilibrium will result although the equilibrium frequencies really only represent relative frequencies of three subpopulations which do not intermate. These frequencies will be:

and

$$
\begin{aligned}
\hat{u} & =\frac{a}{2(h-2 a)} \hat{v}, \\
\hat{v} & =\frac{2(h-2 a)(h-2 b)}{(h-b)(h-2 a)+(h-a)(h-2 b)}, \\
\hat{w} & =\frac{b}{2(h-2 b)} \hat{v},
\end{aligned}
$$

All other cases lead eventually to completely separate homozygotic populations.

If $\alpha<1$ so that some random mating takes place between the genotypes, equations (13)-(15) can be transformed into equations in $p$ and $v$ and equilibrium solutions can be found analogous to those in the previous section. (For details see Falk, 1968). At equilibrium the two relevant equations will be:

$$
T p=a\left[\alpha\left(p-\frac{1}{4} v\right)+(1-\alpha) p^{2}\right]+\frac{1}{2} h\left[\frac{\alpha v}{2}+2(1-\alpha) p(1-p)\right],
$$

and $\quad T v=h\left[\frac{\alpha v}{2}+2(1-\alpha) p(1-p)\right]$,

with $T$ defined in the usual way.

The solution for $v$ in terms of $p$ will be:

where

$$
y=\frac{4 p(1-p) D(p)}{C(p)},
$$

$$
\begin{aligned}
& C(p)=\alpha[\epsilon-p(\epsilon+\delta)] \neq 0, \\
& D(p)=C(p)+p(\epsilon+\delta)-\delta,
\end{aligned}
$$

and $\epsilon$ and $\delta$ are defined as before. Using this expression for $v$, a quadratic in $p$ is again obtained leading to: 


$$
p=\frac{1}{2}+\frac{1}{4}\left\{\eta_{1} \pm \sqrt{\eta_{1}^{2}+4 h k}\right\} \frac{(\delta-\epsilon)}{k}
$$

where

$$
\begin{aligned}
\eta_{1} & =(1+\alpha)(a \delta+b \epsilon)-h(\epsilon+\delta), \\
k & =(1-\alpha)(\epsilon+\delta)(a \delta+b \epsilon) .
\end{aligned}
$$

and

As before, the solution for the special case $a=b$ is seen immediately, and again $\hat{p}=\frac{1}{2}$. Since the solution for $v$ given by (18) does not give a valid result for this special case one must return to the original equations to find $v$. The value of $v$ thus obtained is:

where

$$
\hat{v}=\frac{C_{1}+\sqrt{C_{1}^{2}+2 h(1-\alpha) Q_{1}}}{Q_{1}}
$$

and

$$
C_{1}=\alpha(2 h-a)-(a+h)
$$

$$
Q_{1}=2 \alpha(h-a) \text {. }
$$

Once again the general expression for $\hat{v}$ when $\hat{p} \neq \frac{1}{2}$ is too complicated to be of practical value, but its numerical value can be obtained for particular fitness by using equations (18) and (19).

\section{Discussion}

Equilibrium solutions have been obtained for a model of positive assortative mating where there is differential selection among the genotypes. Both the case of dominance with respect to the characteristic influencing mating choice and the case of no dominance have been considered. In both, if the assortative mating is complete, conditions under which internal equilibria exist, expressions for those equilibria, and local stability properties have been found. Algebraic expressions for a more general model, with a combination of assortative mating and random mating, have been obtained, but conclusions about existence and stability of internal equilibria are not easily obtainable.

In the absence of selection, O'Donald (1960) showed that a stable polymorphism is possible when a fraction of a population is mating assortatively and the remainder at random. With the introduction of selection it is of interest to see under what conditions the stable polymorphism can still be maintained. At one extreme, complete random mating, the condition is already known, namely superior fitness of the heterozygote or heterosis. At the other extreme, complete assortative mating, the conditions are even stricter. When there is no dominance, the heterozygote fitness must be more than twice that of the two homozygotes. When there is dominance, a more subtle relationship between the fitness parameters must hold:

$$
\bar{b}>\frac{h-a}{h-b} .
$$

The genetic interpretation of this inequality is less clear than that of the others, but certain observations provide some clarification. Fig. 1 gives a geometric picture of the quantities involved. If $a>b$ the inequality is always satisfied, for then $\frac{h-a}{h-b}<1$ and it is already required that $\frac{h}{b}>1$ 
(see fig. 1, A). However if $b$ increases and $a$ decreases, there will be a point at which the inequality is reversed (fig. 1, B). Hence the fitness of the $A A$ genotype cannot be greatly depressed relative to the aa genotype and still allow an internal equilibrium to exist.
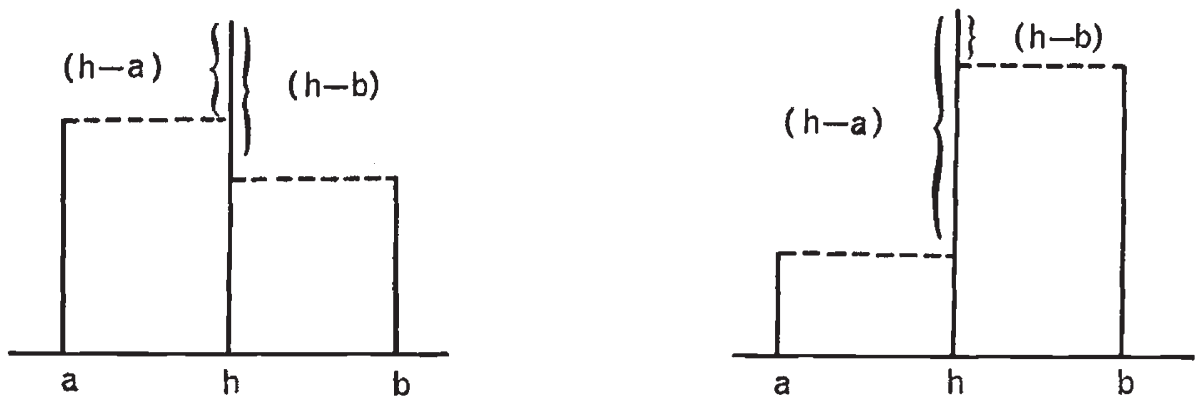

FIG. 1.-Diagrammatic representation of inequalities.

$$
\begin{array}{lc}
\mathrm{A} & \mathrm{B} \\
\frac{h}{b}>\frac{h-a}{h-b} & \frac{h}{b}<\frac{h-a}{h-b}
\end{array}
$$

The more general case, where both random mating and assortative mating are taking place, is very difficult to analyse, and in fact one can say

\begin{tabular}{|c|c|c|c|c|c|c|}
\hline Generation & $v$ & $p$ & $v$ & $p$ & $v$ & $p$ \\
\hline 0 & 0.016 & 0.010 & 0.016 & 0.010 & $0 \cdot 016$ & 0.010 \\
\hline 5 & 0.014 & 0.008 & 0.015 & 0.008 & 0.033 & 0.018 \\
\hline 10 & 0.011 & 0.006 & 0.013 & 0.007 & 0.059 & 0.032 \\
\hline 15 & 0.009 & 0.005 & 0.012 & 0.007 & 0.098 & 0.052 \\
\hline . & . & . & . & . & . & . \\
\hline - & - & . & - & - & · & . \\
\hline 50 & 0.002 & 0.001 & 0.005 & 0.003 & 0.296 & $0 \cdot 159$ \\
\hline . & . & . & . & . & . & . \\
\hline . & . & . & . & . & . & . \\
\hline 100 & 0.000 & 0.000 & 0.002 & $0.0 \dot{0} 1$ & 0.302 & $0 \cdot 162$ \\
\hline . & . & . & . & . & . & . \\
\hline . & . & · & . & $\cdot$ & $\cdot$ & $\cdot$ \\
\hline$\dot{\infty}$ & 0.000 & 0.000 & 0.000 & 0.000 & 0.302 & $0 \cdot \dot{162}$ \\
\hline
\end{tabular}
very little in general. Computer studies can show trends, but no exact

TABLE 2

Partial positive assortative mating with dominance

$a=3, h=33, b=18$.

Therefore $h>a, h>b$ but $\frac{h}{b}>\frac{h-a}{h-b}$.

Note that if $\alpha$ is sufficiently small, the random mating portion of the population will "dominate" and an internal equilibrium is possible.

limitations have yet been obtained. It does seem that for a stable equilibrium heterozygote superiority must obtain. In addition, when there is dominance, if a large proportion of the population is mating assortatively (19) must also 
be satisfied. Table 2 shows three examples of populations in which the fitness parameters do not satisfy (19). The first with $\alpha=1$, becomes homozygous at equilibrium as expected. In the second, $\alpha=0.98$ and the equilibrium population is again homozygous. In the third, $\alpha=0.80$ and the random mating portion of the population is large enough to maintain an internal equilibrium. For other sets of fitness parameters the random mating fraction of the population might have to be even larger in order to maintain an internal equilibrium. There may be a simple relationship between $\alpha, a, h$, and $b$ which provides the information necessary to determine the presence or absence of an internal equilibrium but this has, as yet, not been found.

\section{SUMmary}

1. A model of mixed positive assortative mating and random mating with selection operating on the genotypes at a single, two allele locus is analysed in order to obtain expressions for equilibria and conditions for the local stability of those equilibria whenever possible.

2. The extreme case of complete positive assortative mating is analysed in some detail showing the greater restrictions necessary to maintain a stable polymorphism than are required in a random mating population.

3. Some results for the more general case are presented and the way in which this case fits in between the two extremes is discussed.

Acknowledgment.-This work was supported by grants 2 T01-GM-0039 and HE 09011 from The National Institutes of Health.

\section{Appendix A}

Consider

and

$$
f(u, v)=\frac{a\left(u+\frac{1}{2} v\right)^{2}}{T}=r(u, v) s^{-1}(u, v)
$$

$$
g(u, v)=\frac{h v\left(u+\frac{1}{2} v\right)}{\bar{T}}=t(u, v) s^{-1}(u, v)
$$

where

$$
\begin{aligned}
& r(u, v) \equiv a\left(u+\frac{1}{2} v\right)^{2}, \\
& s(u, v) \equiv \bar{T} \equiv(u+v) \bar{T}=a\left(u+\frac{1}{2} v\right)^{2}+h v\left(u+\frac{1}{2} v\right)+ \\
& \\
&+\frac{b}{4}\left[4 w(u+v)+v^{2}\right],
\end{aligned}
$$

and

$$
t(u, v) \equiv h v\left(u+\frac{1}{2} v\right) .
$$

The partial derivatives of $r, s$ and $t$, with respect to $u$ and $v$, are

$$
\begin{array}{rlrl}
\frac{\partial r}{\partial u} & =2 a\left(u+\frac{1}{2} v\right), & \frac{\partial t}{\partial u} & =h v, \\
\frac{\partial r}{\partial v} & =a\left(u+\frac{1}{2} v\right), & \frac{\partial t}{\partial v} & =h(u+v), \\
\frac{\partial s^{-1}}{\partial u} & =-T_{-2}\left[2 a\left(u+\frac{1}{2} v\right)+h v+b(1-2 u-2 v)\right],
\end{array}
$$


and

$$
\frac{\partial s^{-1}}{\partial v}=-\tilde{T}^{-2}\left[a\left(u+\frac{1}{2} v\right)+h(u+v)+b(1-2 u)\right] .
$$

Now at equilibrium:

and

$$
\begin{aligned}
& \hat{u}=\frac{a M}{D}, \\
& \hat{v}=\frac{2(h-a) M}{D} .
\end{aligned}
$$

Other useful quantities, evaluated at equilibrium are,

$$
\begin{aligned}
\left(\hat{u}+\frac{1}{2} \hat{v}\right) & =\frac{h M}{D}, \\
(\hat{u}+\hat{t}) & =\frac{(2 h-a) M}{D}, \\
1-2 \hat{u} & =\frac{D-2 a M}{D},
\end{aligned}
$$

and

$$
1-2 \hat{u}-2 \hat{v}=\frac{D-2 M(2 h-a)}{D},
$$

where

$$
\mathrm{M} \equiv h^{2}-b(2 h-\mathrm{a}) \text {, }
$$

and

$$
\mathrm{D} \equiv h[(2 h-a)(h-2 b)+b h] \text {. }
$$

The functions $r, s$ and $t$, evaluated at equilibrium, are then:

$$
\begin{aligned}
& r(\hat{u}, \hat{v})=a\left(\frac{h M}{D}\right)^{2}, \\
& t(\hat{u}, \hat{v})=2(h-a)\left(\frac{h M}{D}\right)^{2},
\end{aligned}
$$

and

$$
s^{-1}(\hat{u}, \hat{v})=\frac{D}{h^{2} M} .
$$

Hence the partial derivatives, evaluated at equilibrium are,

$$
\begin{aligned}
r_{u} & =\frac{2 a h M}{D}, \\
r_{v} & =\frac{a h M}{D}, \\
t_{u} & =\frac{2 h(h-a) M}{D}, \\
t_{v} & =\frac{h(2 h-a) M}{D}, \\
s_{u}^{-1} & =-\frac{\mathrm{D}^{2}}{h^{4} M^{2}}\left(2 M^{2}+b D\right),
\end{aligned}
$$


and

$$
s_{v}^{-1}=-\frac{D}{h^{4} M^{2}}\left[2 M\left(h^{2}-a b\right)+b D\right]
$$

where

$$
r_{u}=\left.\frac{\partial r}{\partial u}\right|_{\text {equilibrium, etc. }}
$$

Then

$$
\text { and } \quad \begin{aligned}
g_{v} & =\left(s^{-1} \frac{\partial t}{\partial v}+t \frac{\partial s^{-1}}{\partial v}\right)_{\text {equil. }}= \\
& =\frac{1}{h^{2} D}\left[D\left(2 h^{2}-a h-2 b h+2 a b\right)-4 M(h-a)\left(h^{2}-a b\right)\right]
\end{aligned}
$$

\section{REFERENCES}

BREESE, E. L. 1956. The genetical consequences of assortative mating. Heredity, 10, 323-343. COOCH, F. G., AND BEARDMORE, J. A. 1959. Assortative mating and reciprocal differences in the Blue-Snow Goose complex. Nature, 183, 1833-1834.

FALK, c. T. 1968. Theoretical consequences of assortative mating and selection. Ph.D. Thesis, University of Pittsburgh, (unpublished).

HAYMAN, B. I., AND MATHER, K. 1953. The progress of inbreeding when homozygotes are at a disadvantage. Heredity, 7, 165-184.

KARLIN, s., AND scudo, F. M. 1969. Assortative mating based on phenotype. II. Two autosomal alleles without dominance. Genetics, 63, 499-510.

O'DONALD, P. 1959. Possibility of assortative mating in the Arctic Skua. Nature, 183, 12101211.

O'DONALD, P. 1960. Assortative mating in a population in which two alleles are segregating. Heredity, 15, 389-396.

PARSONS, P. 1962. The initial increase of a new gene under positive assortative mating. Heredity, 17, 267-276.

Parsons, P. 1965. Assortative mating for a metrical characteristic in Drosophila, Heredity, 20, 161-167.

PEARSON, K., AND LEE, A. 1903. On the laws of inheritance in man. I. Inheritance of physical characters. Biometrika, 2, 357-462.

SCUDO, F. M., AND Karlin, s. 1969. Assortative mating based on phenotype. 1. Two alleles with dominance. Genetics, 63, 479-498.

THODAY, J. м. 1959. Effects of disruptive selection. I. Genetic flexibility. Heredity, 13, $187-203$. 